\title{
An Integrated Database of the Ascidian, Ciona intestinalis: Towards Functional Genomics
}

\author{
Yutaka Satou $^{1 \# \star}$, Takeshi Kawashima ${ }^{1 \#}$, Eiichi Shoguchi ${ }^{2}$, \\ Akie Nakayama ${ }^{1}$ and Nori Satoh ${ }^{1,2}$ \\ ${ }^{1}$ Department of Zoology, Graduate School of Science, Kyoto University, \\ Sakyo-ku, Kyoto 606-8502, Japan \\ ${ }^{2}$ CREST, Japan Science Technology Agency, Kawaguchi, \\ Saitama 330-0012, Japan
}

\begin{abstract}
An integrated genome database is essential for future studies of functional genomics. In this study, we update cDNA and genomic resources of the ascidian, Ciona intestinalis, and provide an integrated database of the genomic and CDNA data by extending a database published previously. The updated resources include over 190,000 ESTs $(672,396$ in total together with the previous ESTs) and over 1,000 full-insert sequences (6,773 in total). In addition, results of mapping information of the determined scaffolds onto chromosomes, ESTs from a full-length enriched cDNA library for indication of precise 5'ends of genes, and comparisons of SNPs and indels among different individuals are integrated into this database, all of these results being reported recently. These advances continue to increase the utility of Ciona intestinalis as a model organism whilst the integrated database will be useful for researchers in comparative and evolutionary genomics.
\end{abstract}

Keywords: Ciona intestinalis, ascidian, genome, expressed sequence tag (EST), integrated database

\section{INTRODUCTION}

Recent decoding of genomic sequences of a variety of animals demonstrates their usefulness in a wide range of biological studies. Because the genome sequences themselves do not have any biological meaning, they should be properly annotated to show how many and what kind of genes are encoded. Additionally, especially for functional analyses of the genome, we should more precisely determine gene structures including transcription start sites, exons and introns of each gene, spatial and temporal expression profiles, and phylogenetic annotations of the genes to examine how many paralogs and related genes are encoded in the genome. Much work on genome-wide descriptions has been done for a variety of model organisms. Drosophila melanogaster and Caenorhabditis elegans are leading this field, and a variety of useful annotations continue to be added to their integrated databases, named flybase (Chen et al., 2005) and wormbase (Drysdale et al., 2005), respectively. Within the phylum Chordata, similar work has also begun recently. For example, the $\mathrm{H}$-invita-

\footnotetext{
\# The first two authors contributed equally to this work.

* Corresponding author. Phone:+81-75-753-4095;

Fax :+81-75-705-1113;

Email:yutaka@ascidian.zool.kyoto-u.ac.jp
}

tional database was published recently as a human integrated database (Imanishi et al., 2004).

The ascidian, Ciona intestinalis, is a basal chordate and the seventh animal whose genome sequence has been determined (Dehal et al., 2002). Together with 480,753 ESTs, the genome sequence is an important resource for ascidian biology (Satoh et al., 2003). The genome sequence and basic annotations are browsable in the web site of the Joint Genome Institute (http://genome.jgi-psf.org/ciona4/ ciona4.home.html), and have provided new insights into the origin and evolution of chordates. The genome sequence of Ciona has also revealed that this animal contains less paralogs than those of vertebrates (e.g., Satou et al., 2002b). Because Ciona shares the basic body plan with vertebrates in spite of the simple genome, Ciona provides an efficient experimental system for studying development of the basic body plan common to chordates on a genome-wide scale (Satoh, 2003; Satoh et al., 2003).

For functional analyses of the Ciona genome which aim to explore molecular mechanisms of ascidian development and the origin of the chordates, abundant digitally available cDNA sequences and the availability of their source clones are essential. We have previously made a database based on EST sequences that is accessible via the internet (ghost.zool.kyoto-u.ac.jp) (Satou et al., 2002a). In this data- 
base, all the EST clones are grouped by similarity between their 3'-ESTs into cDNA clusters and therefore each cDNA cluster roughly corresponds to one gene. Various experimental results based on the cDNA clusters have been obtained. For example, we described the spatial expression patterns of 1,000 cDNA clusters each at five different developmental stages and showed the usefulness of the spatial or temporal expression profiles based on EST counts from 12 cDNA libraries for a given gene (Nishikata et al., 2001; Satou et al., 2001; Fujiwara et al., 2002; Kusakabe et al., 2002; Ogasawara et al., 2002; Satou et al., 2002c, 2003a). Full-insert sequences (FISs) of about 5,000 cDNA clones were determined and stored in this database. The source clones have been distributed to researchers all over the world via this database and utilized for their experiments (Satoh, 2004).

Although these two major Ciona web sites share some data with each other, they are not well linked, which is frustrating researchers in functional genomics. After publication of the draft genome of Ciona intestinalis, we have continued to make efforts to enrich the resources for functional genomics, and to update the Ghost web database. Namely, we have mapped the scaffolds to the chromosomes by fluorescent in situ hybridization (FISH) of BAC clones (Shoguchi et al., 2005, submitted). Determination of precise 5'-ends of genes by ESTs from a full-length enriched library has been performed (Satou et al., submitted). A global analysis of polymorphisms has been performed (Kawashima et al., in preparation). Additionally, we have systematically identified almost all transcription factor genes and performed phylogenetic analyses of them as well as genes involved in major signaling pathways such as wnt and TGF- $\beta$ (Chiba et al., 2003; Hino et al., 2003; Kawashima et al., 2003; Sasakura et al., 2003a, 2003b; Satou et al., 2002b, 2003b, 2003c; Wada et al., 2003; Yagi et al., 2003; Yamada et al., 2003). Almost all of their developmental expression profiles have also been described (Imai et al., 2004). For the convenience of researchers, these data should be publicly available via the internet hopefully with the genomic sequence, and therefore we extended the Ghost database to the integrated Ciona intestinalis database, from which all of these data can be retrieved. Together with the ESTs and full insert sequences that we determined in the present study, this integrated database provides a powerful tool for studying functional, comparative and evolutionary genomics.

\section{MATERIALS AND METHODS}

\section{Construction of CDNA libraries and EST sequencing}

We made six cDNA libraries of Ciona intestinalis. Two cDNA libraries from two different stages of juveniles and one cDNA library from the whole body of a mature adult were constructed in pBluescript vector using a commercially available kit (Stratagene). Three libraries from embryos (mixture of eggs to tailbud embryos), the whole body of a mature adult, and the digestive gland were made into pDONR222 using another commercially available kit (Invitrogen). The bacteria containing the libraries were picked into 384 -well plates. The cDNA inserts were amplified by polymerase chain reaction and the amplified cDNA inserts were end-sequenced.

\section{Genome browser interface and computer software used in the} present study

The genome browser interface was constructed based on the generic genome browser package (Stein et al., 2002). The alignments of ESTs and FISs were performed by BLAT (Kent, 2002) and the alignment of whole-genome shotgun reads were performed by BLAST (Altschul et al., 1990).

\section{RESULTS AND DISCUSSION}

\section{Update of the web site}

The web interface providing the cDNA infomation was not changed apart from the addition of a link to the genome browser on each page of cDNA information. The genome browser interface was constructed using the generic genome browser package (Stein et al., 2002). Together with ESTs and FISs of cDNA clones determined since a previous publication (Dehal et al., 2002), all of the available data based on cDNAs are also presented in this genome browser, and each of the cDNA information tracks is tightly linked to the cDNA web resources.

\section{Update of the ESTs and the cDNA full insert sequences}

In previous studies, we made 12 cDNA libraries and obtained 241,519 5'ESTs and 239,234 3'ESTs (480,753 in total) (Dehal et al., 2002). In this study, we newly made cDNA libraries from the digestive gland of the adult, whole adult body except tunic, embryos from the egg to the late tailbud stage and juveniles, and obtained 94,624 5'ESTs and 97,145 3'ESTs (191,769 in total; Table 1; DDBJ/Genbank/EMBL accession numbers, BW318210-BW509978). The combined ESTs cover about $85 \%$ of the predicted gene models (previously covering about 77\%; Dehal et al., 2002). In addition, we collected 11,884 ESTs from the public database from other groups. In addition to the ESTs, we had previously deposited 4,983 FISs of Ciona cDNA clones, to which we added further 1,766 FISs determined in the present study $(6,749$ in total; DDBJ/Genbank/EMBL accession numbers, AK173344-AK175109). We also collected 349 full insert sequences from the public database. All of these sequences are aligned against the genome sequence using the BLAT program (Kent, 2002) and each EST or FIS appearing in the genome browser provides a web link to the corresponding cDNA cluster information, which includes the spatial and temporal expression profiles of the gene as well as its sequence. All of the cDNA clones we used for obtaining the ESTs are freely available for academic purposes.

As previously, we performed gene prediction in the draft genome using the Grail-Exp program (Uberbacher et al., 1996) and this updated cDNA information, which resulted in 19,682 gene models. Accuracy seems to be improved in most cases, which is useful for the ascidian studies. Determining as many cDNA sequences as possible is the only way to obtain accurate gene structures. As described 
Table 1. Summary of ESTs obtained in the previous and present studies.

\begin{tabular}{lrrr}
\hline \multicolumn{1}{c}{ Library } & \multicolumn{1}{c}{ ' EST } & 3' EST & \multicolumn{1}{c}{ Total } \\
\hline ESTs determined in the previous study & & \\
Egg & 29,810 & 29,444 & 59,254 \\
Cleaving embryo & 31,156 & 26,796 & 57,952 \\
Gastrula/Neurula & 23,066 & 23,475 & 46,541 \\
Tailbud embryo & 30,282 & 31,209 & 61,491 \\
Larva & 24,282 & 24,680 & 48,962 \\
Young adult & 28,547 & 29,138 & 57,685 \\
Gonad & 16,048 & 16,239 & 32,287 \\
Testis & 4,655 & 4,717 & 9,372 \\
Endostyle & 2,241 & 2,497 & 4,738 \\
Neural complex & 10,116 & 10,029 & 20,145 \\
Heart & 12,904 & 12,414 & 25,318 \\
Blood cells & 28,412 & 28,596 & 57,008 \\
\hline subtotal & 241,519 & 239,234 & 480,753 \\
& & & \\
ESTs determined in the present study & & \\
Embryo mix & 17,784 & 17,734 & 35,518 \\
Juvenile 1 & 23,914 & 23,897 & 47,811 \\
Juvenile 2 & 3,441 & 3,336 & 6,651 \\
Digestive Gland & 17,582 & 17,808 & 35,390 \\
Mature adult & 31,903 & 34,370 & 66,273 \\
\hline subtotal & 94,624 & 97,145 & 191,643 \\
Total & 336,143 & 336,379 & 672,396 \\
\hline
\end{tabular}

above, we have determined 6,773 cDNA sequences, although some of them are different cDNA clones for the same genes. Accurate gene structure and number will be determined by on-going efforts to determine the cDNA sequences in the future.

\section{Additional useful cDNA and genomic resources ESTs from a full-length cDNA library}

To determine the 5 '-end location of genes precisely, we made a full-length enriched cDNA library from embryos and have started to obtain 5'ESTs from this library (Satou et al., submitted). About $50 \%$ of the ascidian mRNAs contain the spliced-leader, which is added by trans-splicing, and this analysis has revealed gene species making mRNA with the spliced-leader. In addition, because this trans-splicing is used for resolution of polycistronic pre-mRNAs, this analysis has also been able to reveal polycistronic transcription units. At present, 2,079 5'ESTs corresponding to 662 different gene species are available (Fig. 1) and sequencing is ongoing.

\section{Chromosome mapping}

We obtained 17,322 end sequences from 8,661 BAC clones in a previous study (Dehal et al., 2002). These sequences were also aligned against the genome. Using these sequences, the scaffolds have been mapped to chromosomes by FISH of BAC clones (Shoguchi et al., 2005). At present, the mapped scaffolds cover almost $60 \%$ of the whole genome (Shoguchi et al., submitted). In the web site we made in the present study, the results of the mapping of BACs and scaffolds are also shown (Fig. 2).

Polymorphisms revealed by whole-genome shotgun reads from different individuals

Ciona is known to have many polymorphisms, with 15 times as many as those in human (Dehal et al., 2002). The genome project of Ciona intestinalis has been carried out mainly by Japan and the United States. In Japan, two libraries for the whole-genome shotgun sequencing were made from three Japanese individuals, while in the United States several libraries were made from one American individual. Because of the abundance of polymorphisms, the current assembly is basically based on the American individual. We have mapped high-quality regions of the whole-genome shotgun reads in Japan to the current assembly, as well as those from the American one (Fig. 3).

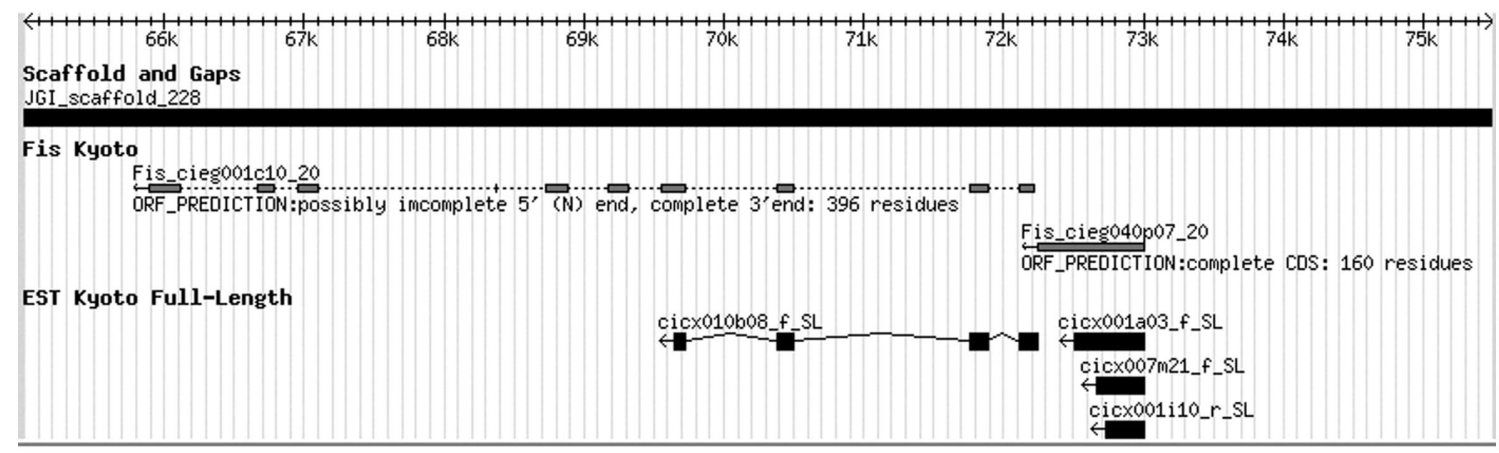

Fig. 1. A screen shot showing 5'-ESTs from a full-length enriched library. In this genomic region, two genes are encoded, which is proven by two full-insert sequences. For each of these two genes, 5'-ESTs from a full-length enriched library were found, indicating the precise $5^{\prime}$-ends of these two genes. The last two letters of the names of 5'-ESTs with the spliced-leader are 'SL'. In this example, these two genes make mRNA with the spliced-leader. 

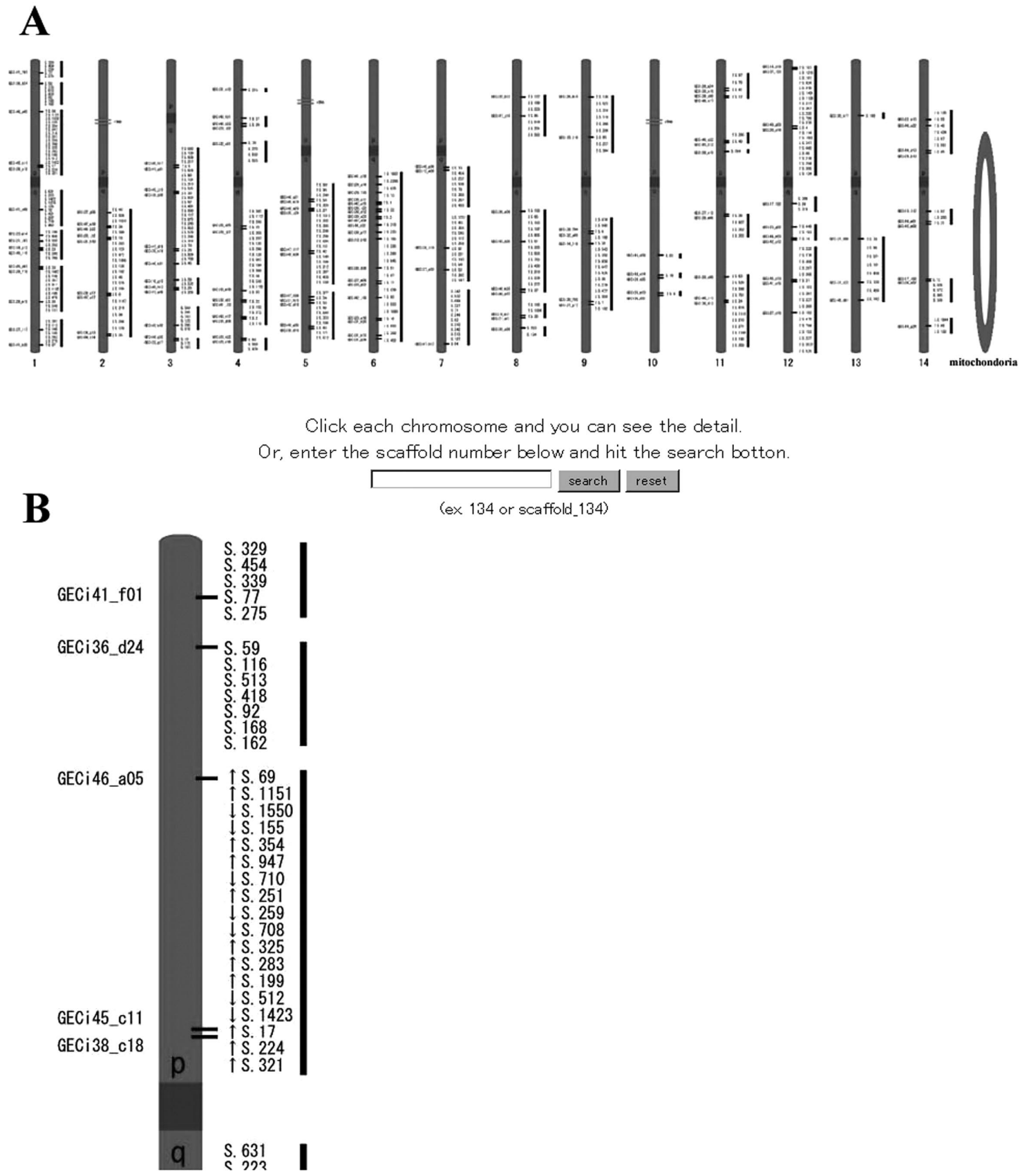

Fig. 2. Screen shots showing the results of mapping of scaffolds onto the chromosomes. (A) A screen shot showing the overview including the search field. (B) A screen shot showing the detailed view of each chromosome.

By this mapping, polymorphisms such as short insertions or deletions (indels) and single nucleotide substitutions (SNPs) can be browsed in the web site. For example, the in silico prediction of the biologically important regions in gene promoters using the polymorphism rate within a species is useful (Boffelli et al., 2004). It has been shown that the polymorphism rate is low in exons and high in introns and in intergenic regions (Kawashima et al., in preparation). Therefore, this alignment shown in the browser will give a powerful tool for predicting biologically important regions of promoters and exons/introns.

\section{Gene expression profiles}

As shown in the previous study, EST counts from each cDNA library for a given gene represent the abundance of the corresponding mRNA at each developmental stage or in each tissue, from which the cDNA libraries were made (Satou et al., 2003a). In the web site constructed based on the cDNA information, we have presented the expression profile of each cDNA cluster (nearly identical with 'gene', which is constructed by comparisons of 3' ESTs with one another). In the present study, the expression profile of each gene model predicted by Grail-Exp with the new dataset, 


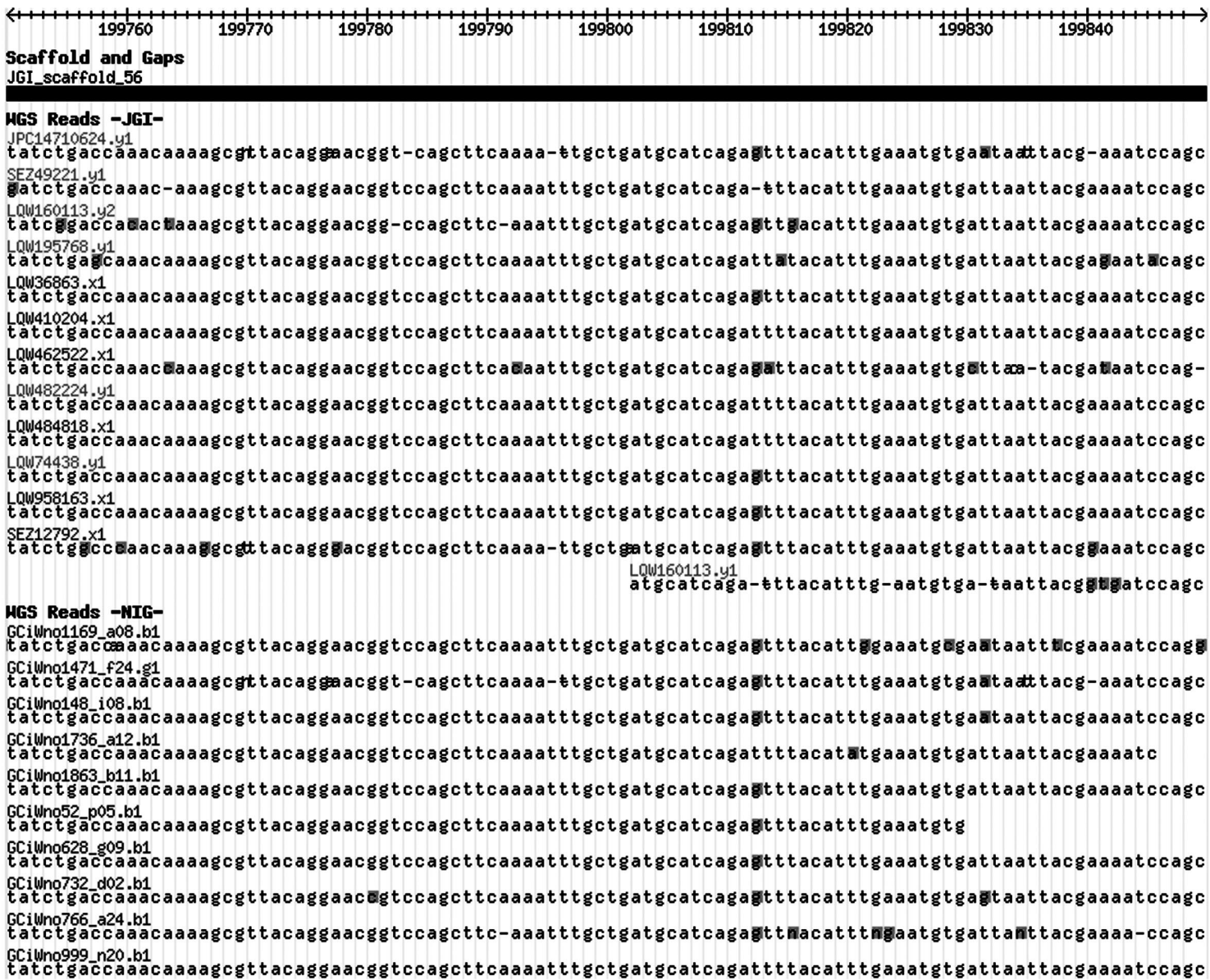

Fig. 3. A screen shot showing an alignment of whole genome shotgun reads from an American individual and three Japanese individuals. Grey boxes indicate nucleotides mismatched with the assembled draft genome sequence.

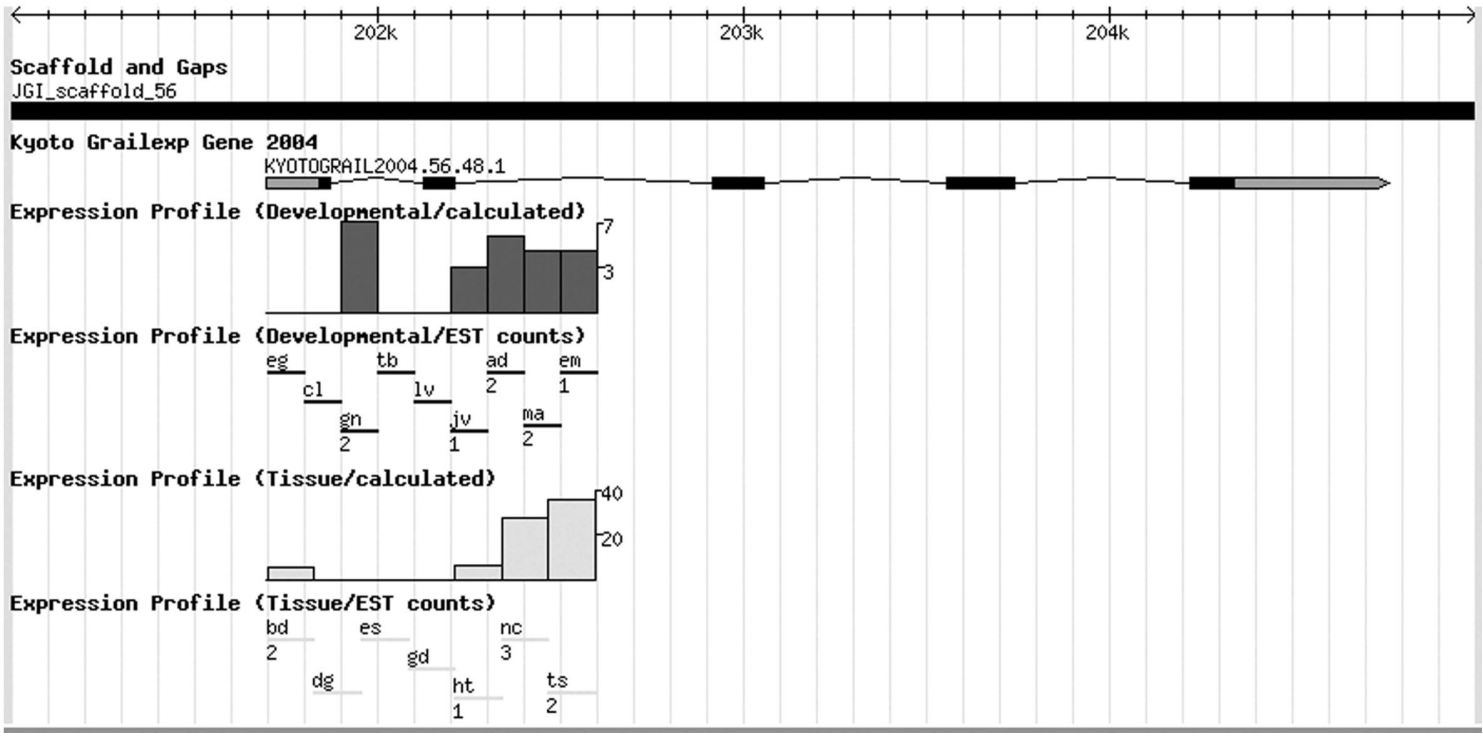

Fig. 4. A screen shot showing a developmental expression profile and a spatial expression profile in an adult. In this example, a gene designated as KYOTOGRAIL2004.56.48.1 is expressed between the gastrula and neurula stages (gn) and then again in juveniles (jv) and young adults (ad), and in a mature adult, this gene is expressed in blood cells (bd), heart (ht), neural complex (nc) and testis (ts). 
which is described above, is shown in the genome browser (Fig. 4).

In addition, we have performed more than 6,000 wholemount in situ hybridization experiments to disclose the spatial expression patterns of developmentally regulated genes (Nishikata et al., 2001; Satou et al., 2001; Fujiwara et al., 2002; Kusakabe et al., 2002; Ogasawara et al., 2002; Imai et al., 2004). These data have also been presented mainly by each cDNA cluster. The web links to these data are added as a track in the genome browser. Therefore, users can easily find the information about when and where the gene is expressed as long as it has been examined.

\section{Microarrays}

We have developed two oligonucleotide-based microarrays (oligomicroarrays) containing about 22,000 probes and 44,000 probes (Agilent technology). These arrays are useful for genome-wide studies of gene expression. Because the probes on the arrays were mapped onto the genome sequence, users can see directly where in the genome the probes of interest are mapped or what kind of genes the probes of interest are. Therefore, users can easily find the expression profile and the relevant information of the gene that the probe of interest indicates. Oligomicroarrays are known to be often severely affected by single-nucleotide substitutions (Hughes et al., 2001). Using mapped whole genome shotgun reads, possible effects of polymorphisms on results of oligomicroarrays can be browsed.

\section{CONCLUSION}

In Ciona intestinalis, lots of effort has been made to improve the genomic information of this basal chordate. Whole genome shotgun reads from a mixture of three individuals other than the one from whom the draft genome was determined correspond to 5 times as many as the genome size, which is useful for analysis of polymorphisms. The determined scaffolds have been mapped to each chromosome by FISH. About 480,000 ESTs and 5,605 FISs have been obtained, and spatial expression profiles of about 1,000 genes in each of 5 developmental stages $(5,000$ genes in total) have been described. Developmental expression profiles of almost comprehensively annotated transcription factor genes and signaling molecule genes in several major pathways have been described. 2,079 5'-ESTs from a full-length enriched library have been obtained. In this report, we determined an additional 191,643 ESTs and 1,766 FISs, and collected ESTs and FISs from the public database. We extended our web-based database, called Ghost, to show all of this information together. In addition to the conventional user interface based on cDNA clustering, we introduced a new user interface based on the generic genome browser. Both interfaces are tightly interlinked with each other. We believe that the resources presented in this integrated database are useful not only for researchers working on ascidians but also for researchers in compara- tive and evolutionary genomics.

\section{ACKNOWLEDGMENTS}

This research was supported by Grants-in-Aid from MEXT, Japan to N.S. and Y.S. (17071020), and CREST to N.S.

\section{REFERENCES}

Altschul SF, Gish W, Miller W, Myers EW, Lipman DJ (1990) Basic local alignment search tool. J Mol Biol 215: 403-410

Boffelli D, Weer CV, Weng L, Lewis KD, Shoukry MI, Pachter L, Keys DN, Rubin EM (2004) Intraspecies sequence comparisons for annotating genomes. Genome Res 14: 2406-2411

Chen N, Harris TW, Antoshechkin I, Bastiani C, Bieri T, Blasiar D, Bradnam K, Canaran P, Chan J, Chen CK et al. (2005) WormBase: a comprehensive data resource for Caenorhabditis biology and genomics. Nucleic Acids Res 33 Database Issue: D383D389

Chiba S, Awazu S, Itoh M, Chin-Bow ST, Satoh N, Satou Y, Hastings KEM (2003) A genomewide survey of developmentally relevant genes in Ciona intestinalis: IX. Genes for muscle structural proteins. Dev Genes Evol 213: 291-302

Dehal P, Satou Y, Campbell RK, Chapman J, Degnan B, De Tomaso A, Davidson B, Di Gregorio A, Gelpke M, Goodstein DM et al. (2002) The draft genome of Ciona intestinalis: insights into chordate and vertebrate origins. Science 298: 2157-2167

Drysdale RA, Crosby MA, Gelbart W, Campbell K, Emmert D, Matthews B, Russo S, Schroeder A, Smutniak F, Zhang $\mathrm{P}$ et al. (2005) FlyBase: genes and gene models. Nucleic Acids Res 33 Database Issue: D390-D395

Fujiwara S, Maeda Y, Shin-i T, Kohara Y, Takatori N, Satou Y, Satoh N (2002) Gene expression profiles in Ciona intestinalis cleavage-stage embryos. Mech Dev 112: 115-127

Hino K, Satou Y, Yagi K, Satoh N (2003) A genomewide survey of developmentally relevant genes in Ciona intestinalis: VI. Genes for Wnt, TGF $\beta$, hedgehog and JAK/STAT signaling pathways. Dev Genes Evol 213: 264-272

Hughes TR, Mao M, Jones AR, Burchard J, Marton MJ, Shannon KW, Lefkowitz SM, Ziman M, Schelter JM, Meyer MR et al. (2001) Expression profiling using microarrays fabricated by an ink-jet oligonucleotide synthesizer. Nat Biotechnol 19: 342-347

Imai KS, Hino K, Yagi K, Satoh N, Satou Y (2004) Gene expression profiles of transcription factors and signaling molecules in the ascidian embryo: towards a comprehensive understanding of gene networks. Development 131: 4047-4058

Imanishi T, Itoh T, Suzuki Y, O'Donovan C, Fukuchi S, Koyanagi $\mathrm{KO}$, Barrero RA, Tamura T, Yamaguchi-Kabata $\mathrm{Y}$, Tanino $\mathrm{M}$ et al. (2004) Integrative annotation of 21,037 human genes validated by full-length cDNA clones. PLoS Biol 2: 859-875

Kawashima T, Tokuoka M, Awazu S, Satoh N, Satou Y (2003) A genomewide survey of developmentally relevant genes in Ciona intestinalis: VIII. Genes for PI3K signaling and cell cycle. Dev Genes Evol 213: 284-290

Kent WJ (2002) BLAT-the BLAST-like alignment tool. Genome Res 12: 656-664

Kusakabe T, Yoshida R, Kawakami I, Kusakabe R, Mochizuki Y, Yamada L, Shin-i T, Kohara Y, Satoh N, Tsuda M et al. (2002) Gene expression profiles in tadpole larvae of Ciona intestinalis. Dev Biol 242: 188-203

Nishikata T, Yamada L, Mochizuki Y, Satou Y, Shin-i T, Kohara Y, Satoh N (2001) Profiles of maternally expressed genes in fertilized eggs of Ciona intestinalis. Dev Biol 238: 315-331

Ogasawara M, Sasaki A, Metoki H, Shin-i T, Kohara Y, Satoh N, Satou $Y(2002)$ Gene expression profiles in young adult Ciona 
intestinalis. Dev Genes Evol 212: 173-185

Sasakura Y, Yamada L, Takatori N, Satou Y, Satoh N (2003a) A genomewide survey of developmentally relevant genes in Ciona intestinalis: VII. Molecules involved in the regulation of cell polarity and actin dynamics. Dev Genes Evol 213: 273-283

Sasakura Y, Shoguchi E, Takatori N, Wada S, Meinertzhagen IA, Satou Y, Satoh N (2003b) A genomewide survey of developmentally relevant genes in Ciona intestinalis: X. Genes for cell junctions and extracellular matrix. Dev Genes Evol 213: 303313

Satoh N (2003) The ascidian tadpole larva: Comparative molecular development and genomics. Nature Rev Genet 4: 285-295

Satoh N (2004) Genomic resources for ascidians: Sequence/expression databases and genome projects. In "Methods in Cell Biology: Experimental analysis of the development of sea urchins and other non-vertebrate deuterostomes Vol 74" Ed by CA Ettensohn et al., Elsevier Inc., pp 759-774

Satoh N, Satou Y, Davidson B, Levine M (2003) Ciona intestinalis: an emerging model for whole-genome analyses. Trends Genet 19: $376-381$

Satou Y, Takatori N, Yamada L, Mochizuki Y, Hamaguchi M, Ishikawa H, Chiba S, Imai K, Kano S, Murakami SD et al. (2001) Gene expression profiles in Ciona intestinalis tailbud embryos. Development 128: 2893-2904

Satou Y, Yamada L, Mochizuki Y, Takatori N, Kawashima T, Sasaki A, Hamaguchi M, Awazu S, Yagi K, Sasakura Y et al. (2002a) A cDNA resource from the basal chordate Ciona intestinalis. Genesis 33: 153-154

Satou Y, Imai KS, Satoh N (2002b) Fgf genes in the basal chordate Ciona intestinalis. Dev Genes Evol 212: 432-438

Satou Y, Takatori N, Fujiwara S, Nishikata T, Saiga H, Kusakabe T, Shin-i T, Kohara Y, Satoh N (2002c) Ciona intestinalis cDNA projects: expressed sequence tag analyses and gene expression profiles during embryogenesis. Gene 287: 83-96

Satou Y, Kawashima T, Kohara Y, Satoh N (2003a) Large scale EST analyses in Ciona intestinalis: Its application as Northern blot analyses. Dev Genes Evol 213: 314-318

Satou Y, Imai KS, Levine M, Kohara Y, Rokhsar D, Satoh N (2003b) A genomewide survey of developmentally relevant genes in Ciona intestinalis: I. Genes for bHLH transcription factors. Dev Genes Evol 213: 213-221

Satou Y, Sasakura Y, Yamada L, Imai KS, Satoh N, Degnan B (2003c) A genomewide survey of developmentally relevant genes in Ciona intestinalis: V. Genes for receptor tyrosine kinase pathway and Notch signaling pathway. Dev Genes Evol 213: 254-263

Shoguchi E, Kawashima T, Nishida-Umehara C, Matsuda Y, Satoh $\mathrm{N}$ (2005) Molecular cytogenetic characterization of Ciona intestinalis chromosomes. Zool Sci 22: 511-516

Stein LD, Mungall C, Shu S, Caudy M, Mangone M, Day A, Nickerson E, Stajich JE, Harris TW, Arva A et al. (2002) The generic genome browser: a building block for a model organism system database. Genome Res 12: 1599-1610

Uberbacher EC, Xu Y, Mural RJ (1996) Discovering and understanding genes in human DNA sequence using GRAIL. Methods Enzymol 266: 259-281

Wada S, Tokuoka M, Shoguchi E, Kobayashi K, Di Gregorio A, Spagnuolo A, Branno M, Kohara Y, Rokhsar D, Levine M et al. (2003) A genomewide survey of developmentally relevant genes in Ciona intestinalis: II. Genes for homeobox transcription factors. Dev Genes Evol 213: 222-234

Yagi K, Satou Y, Mazet F, Shimeld SM, Degnan B, Rokhsar D, Levine M, Kohara Y, Satoh N (2003) A genomewide survey of developmentally relevant genes in Ciona intestinalis: III. Genes for Fox, ETS, nuclear receptors and NFKB. Dev Genes Evol 213: $235-244$

Yamada L, Kobayashi K, Degnan B, Satoh N, Satou Y (2003) A genomewide survey of developmentally relevant genes in Ciona intestinalis: IV. Genes for HMG transcriptional regulators, bZip and GATA/Gli/Zic/Snail. Dev Genes Evol 213: 245-253

(Received June 22, 2005 / Accepted June 30, 2005) 